\title{
Preparation and Characterization of Magnetic Nanoparticles
}

\author{
I. Khmara ${ }^{a, b}$, M. Kubovcikova ${ }^{a, *}$, M. KonerackA ${ }^{a}$, B. Kalska-Szostko ${ }^{c}$, V. Zavisova $^{a}$, \\ I. $\operatorname{Antal}^{a}$, M. RAJnAK ${ }^{a}$, Z. DAnKOVA ${ }^{d}$, V. KAVECANSKY $^{a}$, M. OMAStOVA $^{e}$ \\ AND P. KOPCANSKY ${ }^{a}$ \\ ${ }^{a}$ Institute of Experimental Physics, Slovak Academy of Sciences, Watsonova 47, 04001 Kosice, Slovakia \\ ${ }^{b}$ Institute of Physics, Faculty of Sciences, P. J. Safarik University, Park Angelinum 9, 04154 Kosice, Slovakia \\ ${ }^{c}$ Institute of Chemistry, University of Bialystok, ul. Ciolkowskiego 1K, 15-245 Bialystok, Poland \\ ${ }^{d}$ Institute of Geotechnics, Slovak Academy of Sciences, Watsonova 45, 04001 Kosice, Slovakia \\ ${ }^{e}$ Polymer Institute, Slovak Academy of Sciences, Dubravska cesta 9, 84541 Bratislava, Slovakia
}

\begin{abstract}
The magnetic nanoparticles with core diameter $10 \mathrm{~nm}$ were modified by poly-L-lysine to bind antibody for cancer cell detection. Prepared biocompatible magnetic fluid (MFPLL) was characterized by dynamic light scattering method to obtain the particle size distribution. The microstructure of the MNPs and MFPLL samples were studied by transmission electron microscopy, X-ray diffraction and Mössbauer spectroscopy. Magnetic properties of the samples were measured by SQUID magnetometer and superparamagnetic behaviour of the samples was confirmed.
\end{abstract}

DOI: 10.12693/APhysPolA.133.704

PACS/topics: 47.65.Cb, 61.05.Qr, 61.05.cp

\section{Introduction}

In recent years, magnetic nanoparticles (MNPs) have been widely used in technical and biomedical applications. For technical applications, MNPs are generally coated with oleic acid monolayer, the molecules of which prevent aggregation of particles and form a long-term stabilization without affecting the magnetic properties (superparamagnetism) of MNPs [1]. For biomedical applications a special place among all the known MNPs is taken by iron oxide (magnetite, maghemite and hematite), which in terms of magnetization is one of the important magnetic iron oxides. Besides strong magnetism, magnetite shows low toxicity and biocompatibility, makes it quite attractive for therapeutic and diagnostic applications.

The difficulties in MNPs preparation are related to the fact that nanoparticles that have a large ratio of surface area to volume tend to agglomerate. This problem can only be solved out with the decrease of surface energy of particles, which will provide magnetic fluid (MF) stability and prevent agglomeration [2] for example by suitable coating or modification of the MNPs surface.

In our work, poly-L-lysine (PLL) was used for stabilization and amino-modification of the iron oxide nanoparticles. Poly-L-lysine was selected because of good impact of its monomer - lysine on the human body. The main functional groups in lysine are carboxyl group and two amino groups. Due to carboxyl group of molecule, lysine is easily attached to the surface of MNPs and free amino groups can be attached to the antibody [3].

*corresponding author; e-mail: kubovcikova@saske.sk

\section{Experiment}

Poly-L-Lysine (PLL) with molecular weight 150,000-300,000 $\mathrm{g} / \mathrm{mol}$, ferric chloride hexahydrate $\left(\mathrm{FeCl}_{3} \cdot 6 \mathrm{H}_{2} \mathrm{O}\right)$, ferrous sulphate heptahydrate $\left(\mathrm{FeSO}_{4} \cdot 7 \mathrm{H}_{2} \mathrm{O}\right)$ and ammonium hydroxide $\left(\mathrm{NH}_{4} \mathrm{OH}\right)$ were purchased from Sigma Aldrich.

Iron oxide $\left(\mathrm{Fe}_{3} \mathrm{O}_{4}\right)$ MNPs were prepared by coprecipitation method of ferric and ferrous salts with a molar ratio 2:1. Under vigorous stirring, ammonium hydroxide solution was added into the flask with mixture of water solution of $\mathrm{Fe}^{3+}$ and $\mathrm{Fe}^{2+}$, resulting in immediate magnetite formation in the form of a black precipitate. Obtained MNPs were washed four times (non-sonicated MNPs), subsequently they were put into a glass vial with defined volume of water and sonicated for $5 \mathrm{~min}$ at $70 \%$ power (Branson digital sonifier) (sonicated MNPs).

The next step was MNPs modification with PLL. The suspension of MNPs was mixed with PLL solution $(0.1 \%)$ at the PLL $/ \mathrm{Fe}_{3} \mathrm{O}_{4}$ weight ratio 3 . The mixture was sonicated for 5 min at $70 \%$ power in the ice bath. In order to increase the MNPs concentration, magnetic suspension modified by PLL (MFPLL) was centrifuged at 100,000 g during $1 \mathrm{~h}$ at $4{ }^{\circ} \mathrm{C}$. Having removed supernatant from centrifuged samples, the sediment was thoroughly dispersed in ultrapure water and obtained MFPLL was collected into the flask.

Physical-chemical characterization of both sonicated and non-sonicated MNPs and MFPLL was determined by different methods. In order to determine the size and morphology of MNPs and MFPLL samples, transmission electron microscopy (TEM, JEOL 2100F microscope operated at $200 \mathrm{kV}$ ) was used.

Surface properties of studied samples were determined from the adsorption and desorption isotherms measured with the NOVA 1200e Surface Area \& Pore Size Analyzer (Quantachrome Instruments, USA) by the method 
of physical adsorption of nitrogen at $-196^{\circ} \mathrm{C}$. The values of specific surface area $\left(S_{B E T}\right)$ were determined using BET isotherm. The values of external surface $\left(S_{\text {ext }}\right)$ and volume of micropores $\left(V_{\text {micro }}\right)$ were calculated from the $t$-plot method. The pore size distribution was obtained from the desorption isotherm by BJH method.

The particle size distribution was measured by dynamic light scattering (DLS) technique (Zetasizer NanoZS, Malvern, UK), which measures the velocity of particles Brownian motion. The stability of the samples was studied by the measuring of Zeta potential using laser Doppler velocity. The magnetic properties measurements were conducted by SQUID magnetometer (Quantum Design MPMS 5XL). X-ray diffraction (XRD, Rigaku, Ultima $\mathrm{IV}, \mathrm{Cu} \mathrm{K} \mathrm{K}_{\alpha}$ radiation) technique was used for the qualification of magnetite. Mössbauer spectra were measured at room temperature by spectrometer working in constant acceleration mode with a $\mathrm{Co}^{57}$ isotope source.

\section{Results and discussion}

The core diameter of the MNPs was observed by TEM. It was proved that the size of all samples is very similar and close to $10 \mathrm{~nm}$ (Fig.1 a-c).

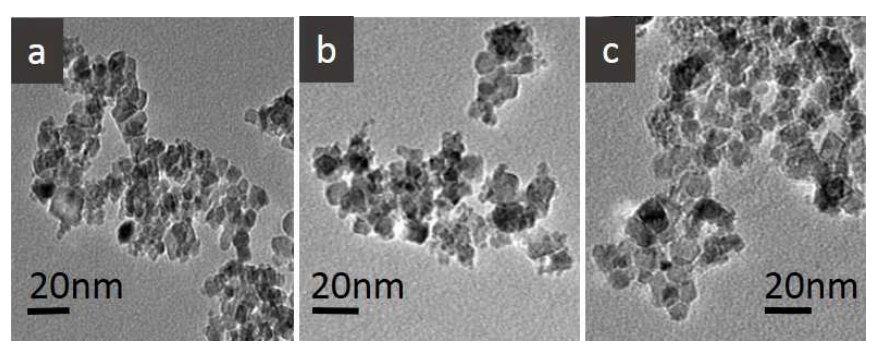

Fig. 1. TEM image of sonicated MNPs (a); nonsonicated MNPs (b); MFPLL (c).

The measured adsorption and desorption isotherms of both samples create the hysteresis loop in the range of relative pressure $p / p_{0}>0.7$ (Fig. 2). The desorption branch is closed continuously without the expressive jump on the branch. The capillary condensation is generally associated with the presence of mesopores in the studied structures. Whereas the iron oxide particles are non-porous and they have tendency to create agglomerates, the presence of hysteresis loop pointed at the mesopore structure formation between the iron oxide particles. The value of adsorbed volume is higher for the non-sonicated sample than for sonicated, also the value of specific surface area is higher, but the difference is not so significant. The values of external surface are almost equal to $S_{B E T}$ values; none or no significant presence of micropores is expected. The non-sonicated sample showed broader distribution of mesopores (from the diameter $5 \mathrm{~nm}$ up to $45 \mathrm{~nm}$ ) and its distribution curve indicates the presence of larger pores - macropores. The sonicated sample showed narrow distribution of pores with the maximum $14.5 \mathrm{~nm}$ without the presence of macropores (Fig. 2 inset). Mean MNPs diameters obtained by different methods are summarized in Table I.

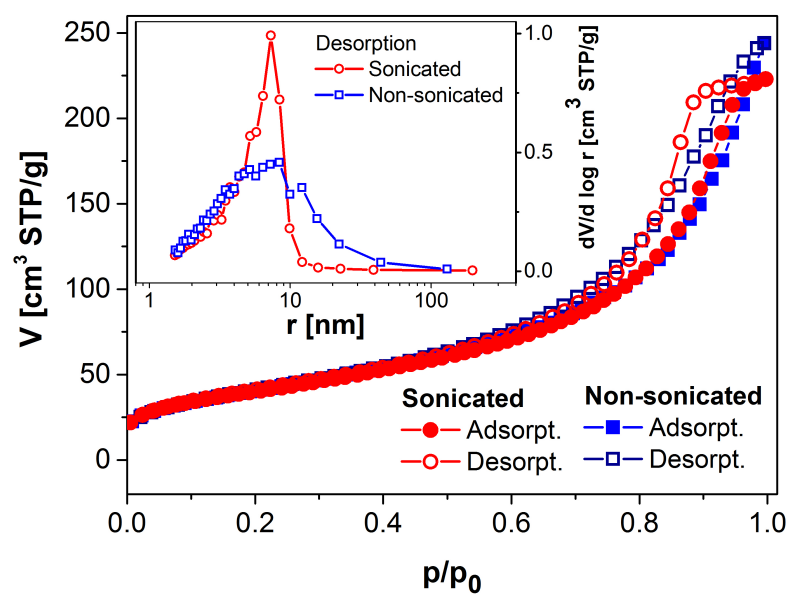

Fig. 2. Adsorption and desorption isotherms of sonicated MNPs and non-sonicated MNPs; inset: pore size distribution curves of sonicated MNPs and nonsonicated MNPs.

TABLE I

Physical properties of non-sonicated MNPs, sonicated MNPs and MFPLL.

\begin{tabular}{c|c|c|c|c|c|c|c}
\hline \hline \multirow{2}{*}{ Sample } & $D_{B E T}$ & $D_{X R D}$ & $D_{M A G}$ & $D_{D L S}$ & $\begin{array}{c}Z \text {-pot } \\
{[\mathrm{mV}]}\end{array}$ & $\begin{array}{c}\mathrm{M}_{S} \\
{\left[\mathrm{emu} / \mathrm{g}_{\mathrm{Fe}}\right.}\end{array}$ \\
\cline { 2 - 5 } & \multicolumn{5}{|c|}{$[\mathrm{nm}]$} & 20 & 77.7 \\
sonicated & 10.2 & $3.8(2)$ & 12.0 & 54 & 20 & 74.8 \\
non-son. & 10.1 & $5.0(3)$ & 12.0 & 190 & 34 & 7.5 \\
MFPLL & - & - & 12.8 & 124 & 43 & 7.5
\end{tabular}

Hydrodynamic particle size distribution measured by DLS method revealed that $z$-average of MNPs decreased from 190 to $54 \mathrm{~nm}$ after sonication process. Regarding zeta potential measurement, obtained results indicated fine colloidal stability of studied MFs (see Table I).

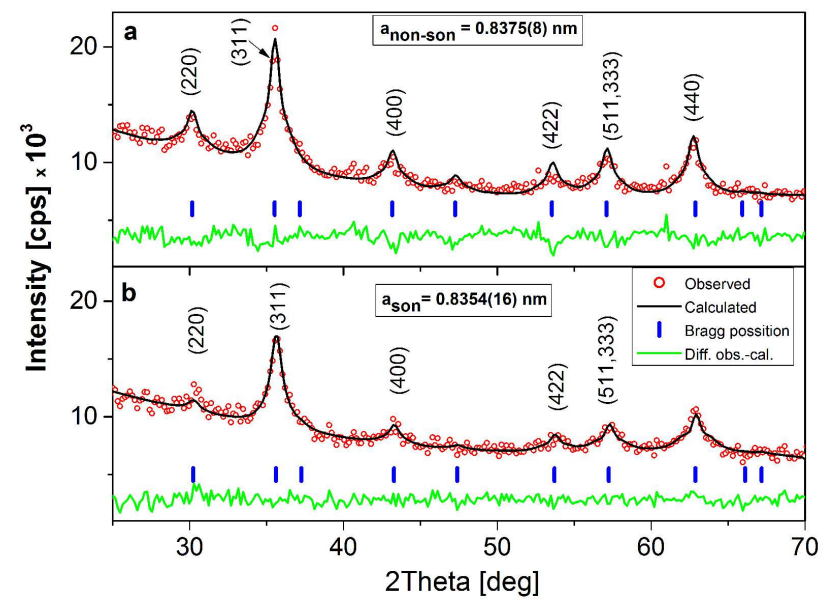

Fig. 3. Rietveld plot of the non-sonicated (a) and sonicated (b) MNPs.

The synthesized MNPs were studied by X-ray diffraction method. The XRD patterns of the sonicated and non-sonicated MNPs (Fig. 3) were fitted by the Rietveld method based on the structural model of mag- 
netite. However, the phase identification of magnetite and maghemite by the conventional X-ray diffraction method is rather complicated as both have the same cubic structure and their lattice parameters are almost identical. Furthermore, the powder samples showed rather small average grain size. Consequently, the diffraction peaks were broadened and the resolution of the experiment significantly decreased. Mean linear diameters $D_{X R D}$ of the coherent region estimated by X-ray diffraction (Halder-Wagner method) are summarized in Table I.

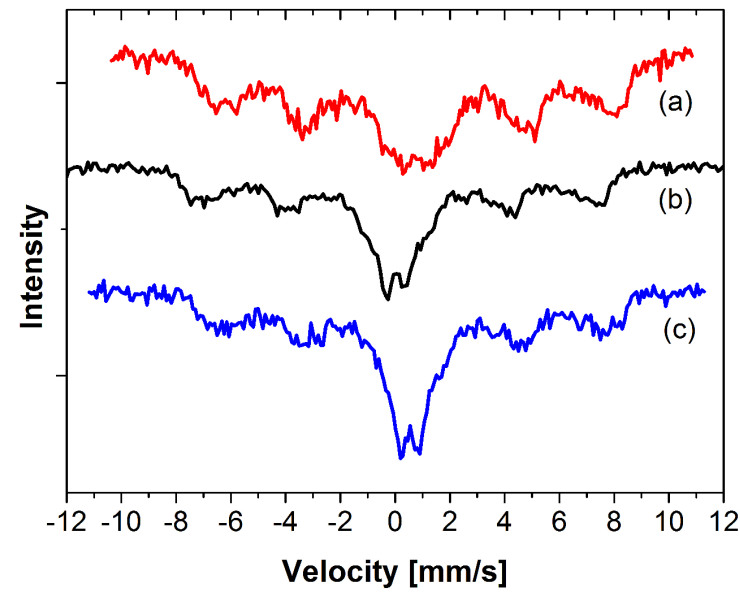

Fig. 4. Mössbauer spectra of non-sonicated MNPs (a); sonicated MNPs (b); MFPLL (c) measured at room temperature.

Qualitative analysis of Mössbauer spectra suggests that sonication and surface modification by PLL has an influence on core composition or interparticle interaction (Fig. 4). Changes observed as increase of central doublet intensity in comparison to sextet contribution can be a result of both. Such modifications of the spectra due to interparticle interaction were observed earlier [4, 5].

The magnetization curves of MNPs and MFPLL measured at room temperature are shown in Fig. 5. Magnetic measurements confirmed superparamagnetic behaviour of the samples at room temperature.

The saturation magnetization and the magnetic core diameter of MNPs calculated from magnetization curves using the formula introduced by Chantrell [6] are shown in Table I. It was found that neither sonication nor modification has an influence on the magnetic core diameter of the MNPs.

\section{Conclusion}

The magnetic $\mathrm{Fe}_{3} \mathrm{O}_{4}$ nanoparticles were prepared by the co-precipitation method. Properties of nonsonicated, sonicated MNPs and MNPs coated by PLL were compared. Both samples (MNPs before and after sonication) showed similar values of specific surface area. The tendency of iron oxide particles to create agglomerates led to creation of mesopore structure between the iron oxide particles. The sonication process has refined the agglomerates. The sonicated sample did not contain the macropores, and the distribution of pores was

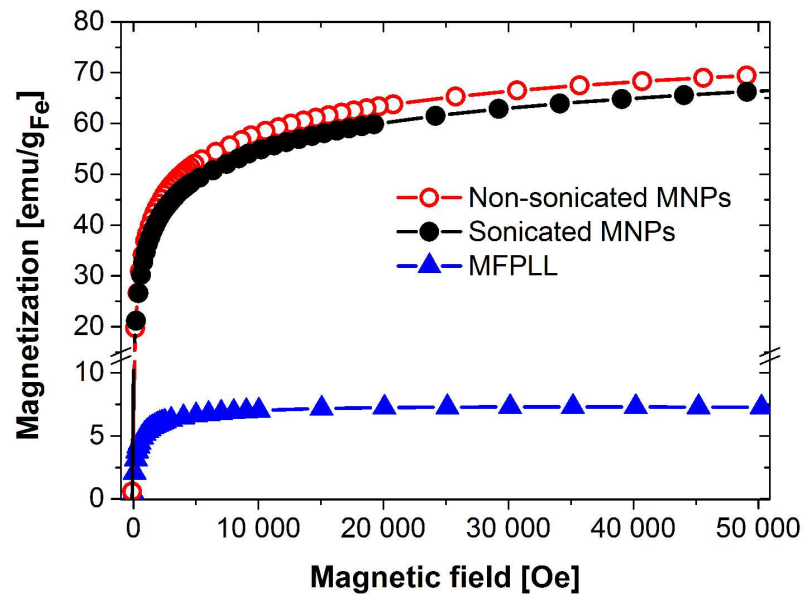

Fig. 5. Magnetization vs. applied magnetic field for non-sonicated MNPs, sonicated MNPs and MFPLL.

narrower with comparison with non-sonicated sample. Sonication process has an influence on MNPs chemical composition (probable oxidation to maghemite, which is indicated by Mössbauer spectra changes). Measuring of magnetic properties of the samples showed their superparamagnetic behaviour at room temperature. It was found that sonication has a significant influence neither on the core diameter nor saturation magnetization of the MNPs. Based on these results sonicated MNPs were chosen for PLL functionalization for further binding of antibody for cancer cell detection.

\section{Acknowledgments}

This work was supported by Ministry of Education Agency for Structural Funds of EU in the frame of Projects 26220120033, 26220220186, Slovak Research and Development Agency under Contract nos. APVV-14-0120, APVV-14-0932 and APVV-15-0453 and COST TD1402 Radiomag and EU founds via projects POPW.01.03.00-20-034/09-00 and POPW.01.03.00-20004/11-00.

\section{References}

[1] S. Campelj, D. Makovec, M. Drofenik, J. Phys.: Condens. Matter 20, 1 (2008).

[2] D. K. Kim, Y. Zhang, W. Voit, K. V. Rao, M. Muhammed, J. Magn. Magn. Mater. 225, 30 (2001).

[3] I. Khmara, M. Koneracka, M. Kubovcikova, V. Zavisova, I. Antal, K. Csach, P. Kopcansky, I. Vidlickova, L. Csaderova, S. Pastorekova, M. Zatovicova, J. Magn. Magn. Mater. 427, 114 (2017).

[4] B. Kalska-Szostko, M. Zubowska, D. Satula, Acta Phys. Pol. A 109, 365 (2006).

[5] D. Satula, B. Kalska-Szostko, K. Szymanski, L. Dobrzynski, J. Kozubowski, Acta Phys. Pol. A 114, 1615 (2008).

[6] R.W. Chantrell, J. Popplewell, S.W. Charles, IEEE Trans Mag 14, 975-977 (1978). 